\title{
A NOTE ON SEPARATION AXIOMS WEAKER THAN $T_{1}$
}

\author{
S. M. ROBINSON and Y. C. WU
}

(Received 23 June 1967)

\section{Introduction}

In $[\mathbf{l}]$, Aull and Thron introduce several separation axioms between $T_{0}$ and $T_{1}$. In particular, they define a space $X$ to be $T_{D}$ if for each $x \in X$, $\{x\}^{\prime}$ is a closed set.

On page 37 of their paper, Aull and Thron ask whether there is a separation axiom weaker than $T_{1}$ which when combined with normality implies $T_{4}$. In [2], Thron asks whether a product of $T_{D}$ spaces is again a $T_{D}$ space. In the first section of this note, we answer both of these questions: the first question admits a positive solution, while the second conjecture is never true in the infinite case. In the second section of this paper, we investigate a heirarchy of separation axioms weaker than $T_{D}$.

\section{1}

The answer to the second question is contained in the following theorem.

THEOREM 1. If $\left\{X_{i}: i \in I\right\}$ is an infinite collection of $T_{D}$ spaces which are not $T_{1}$, then $\Pi\left\{X_{i}: i \in I\right\}$ is not a $T_{D}$ space.

Proof. Since each $X_{i}$ is not a $T_{1}$ space, there exists an $\alpha \in \Pi\left\{X_{i}: i \in I\right\}$ such that for each $i$, the derived set of the $i$-th projection of $\alpha,\left\{\pi_{i}(\alpha)\right\}^{\prime} \neq \emptyset$. Let $\left.Y=\Pi \overline{\left\{\pi_{i}(\alpha)\right.}=i \in I\right\}$ and let $\{\alpha\}^{\prime *}$ denote the derived set of $\alpha$ in the relative topology. Since the property of being $T_{D}$ is inherited by subspaces, it suffices to show that $\{\alpha\}^{* *}$ is not closed in $Y$. We first show that $\{\alpha\}$ is not open in $Y$. To do this, we observe first that if 0 is a basic open neighborhood of $y$ in $Y, 0=Y \cap \Pi\left\{0_{i}: i \in I\right\}$, where each $0_{i}$ is an open neighborhood of $\pi_{i}(y)$ in $X_{i}$ and $0_{i}=X_{i}$ for almost all $i \in I$. Since $I$ is infinite, there exists a non-empty subset $I^{\prime} \subset I$ such that $0_{i}=X_{i}$ for all $i \in I^{\prime}$. Let 0 be such a basic neighborhood of $\alpha$. Because our choice of $\alpha$ implies that $\overline{\pi_{i}(\alpha)}-\pi_{i}(\alpha) \neq \emptyset$ for $i \in I$, we may select $\beta \in Y$ such that $\pi_{i}(\beta)=\pi_{i}(\alpha)$ for $i \in I-I^{\prime}$ and $\pi_{i}(\beta) \neq \pi_{i}(\alpha)$ for $i \in I^{\prime}$. Clearly, $\beta \in 0$; hence $\{\alpha\}$ is not open.

1 Presented to the American Mathematical Society on March 6, 1967. 
Let $\rho \in Y-\{\alpha\}$ and let 0 be a basic open neighborhood of $\rho$. The observation that $\pi_{i}(\rho) \in \overline{\pi_{i}(\alpha)}$ yields that $\pi_{i}(\alpha) \in \pi_{i}(0)$ for $i \in I$. Therefore $\rho \in\{\alpha\}^{\prime *}$. Hence $\{\alpha\}^{*}=Y-\{\alpha\}$ and so $\{\alpha\}^{*}$ is not closed.

The following corollary shows that there is no possibility of introducing a separation axiom between $T_{D}$ and $T_{1}$ that is inherited by arbitrary products.

Corollary. Let $X$ be a $T_{D}$ space. Then, an arbitrary product of $X$ is a $T_{D}$ space if and only if $X$ is $T_{1}$.

We shall show subsequently that there is no separation axiom between a much weaker axiom than $T_{D}$ and $T_{1}$ that is inherited by arbitrary products.

Definition. $X$ is a strong $T_{D}$ space if for each $x \in X,\{x\}^{\prime}$ is either empty or is a union of a finite family of non-empty closed sets, such that the intersection of this family is empty.

For example, $X=\{a, b, c\}$ with topology $\{\emptyset,\{a\},\{a, b\},\{a, c\}, X\}$ is a strong $T_{D}$ space which is not $T_{1}$.

Definition. $X$ is a strong $T_{0}$ space provided that for each $x \in X,\{x\}^{\prime}$ is either empty or a union of non-empty closed sets, such that the intersection of this union is empty and at least one of the non-empty members is compact.

THEOREM 2. A normal space $X$ which is either strong $T_{0}$ or strong $T_{D}$ is $T_{4}$.

Proof. It suffices to show that $X$ is $T_{1}$. Suppose $X$ is strong $T_{0}$ and normal, and consider $x \in X$. We claim that $\{x\}^{\prime}=\emptyset$. For if not, $\{x\}^{\prime}=\bigcup\left\{C_{i}: i \in I\right\}$, where each $C_{i}$ is non-empty. Let $C_{1}$ be a compact set in this collection and let $\mathscr{I}=\left\{J \subset I: \cap\left\{C_{i}: i \in J\right\} \cap C_{1} \neq \emptyset\right\}$. $\mathscr{I}$ is a non-empty collection and is partially ordered by set inclusion; furthermore, the compactness of $C_{1}$ guarantees that $\mathscr{I}$ is inductive. Thus, Zorn's lemma assures a maximal element $I^{*}$ of $\mathscr{I}$. Let $F=\cap\left\{C_{i}: i \in I^{*}\right\}$; clearly, there exists an $i_{0} \in I$ such that $C_{i_{0}} \cap F=\emptyset$. But normality implies $C_{i_{0}}$ and $F$ can be separated by disjoint open sets, $0_{1}$ and $0_{2}$. Because $C_{i_{0}}$ and $F$ are non-empty subsets of $\{x\}^{\prime}, 0_{1}$ and $0_{2}$ meet $\{x\}^{\prime}$ and hence $x$. This is impossible since $0_{1} \cap 0_{2}=\emptyset$.

The proof for the strong $T_{D}$ case is similar and does not invoke any form of the axiom of choice.

REMARK. In the definition of strong $T_{0}$ space, we cannot omit the compactness condition and still obtain the theorem. For example, let $N$ be the natural numbers and let the topology be

$$
\{\emptyset, N,\{1\},\{1,2\},\{1,2,3\}, \cdots,\{1,2,3, \cdots, n\}, \cdots\} .
$$

For each 


$$
\begin{aligned}
n \in N,\{n\}^{\prime} & =\{n+1, n+2, \cdots\} \\
& =\cup\{\{n+j, n+j+1, n+j+2, \cdots\}: j=1,2, \cdots\} \\
& =\cup\left\{C_{n, j}: j=1,2, \cdots\right\},
\end{aligned}
$$

and we notice that $\cap\left\{C_{n, j}: j=1,2, \cdots\right\}=\emptyset$. However, this space is normal and not $T_{\mathbf{4}}$.

In this section we shall discuss separation axioms weaker than $T_{D}$ and characterize spaces enjoying one of these separation properties.

Definition. Let $m$ be an infinite cardinal. $X$ is a $T^{(m)}$ space provided that for each $x \in X,\{x\}=F \cap\left(\cap\left\{0_{i}: i \in I\right\}\right)$, where $F$ is closed, each $0_{i}$ is open, and card $(I)=m$.

THEOREM 3. The following statements are equivalent.

i. $X$ is a $T^{(m)}$ space.

ii. For each $x \in X,\{x\}^{\prime}$ is an m-fold union of closed sets.

iii. For each $A \subseteq X$, such that card $(A) \leqq m, A^{\prime}$ is an m-fold union of closed set.

PROOF. i $\rightarrow$ ii. $\quad\{x\}^{\prime}=\overline{\{x\}}-\{x\}=\overline{\{x\}}-\left(F \cap\left(\cap\left\{0_{i}: i \in I\right\}\right)\right) . \quad$ Upon noting that $\overline{\{x\}} \subset F$ and applying the De Morgan laws, we obtain $\{x\}^{\prime}=\cup\left\{X-0_{i}: i \in I\right\}$.

ii $\rightarrow$ iii. Let $A=\left\{x_{i}: \in I\right\}$, where card $(I) \leqq m$. Let $C$ denote the set of $\omega$-limit points of $A$; clearly, $C$ is closed. Thus,

$$
A^{\prime}=C \cup\left(\cup\left\{\left\{x_{i}\right\}^{\prime}: x_{i} \in A\right\}\right),
$$

for $A^{\prime}$ certainly contains the right hand side and if $x \in A^{\prime}-C$, then $x \in\left\{x_{i}\right\}^{\prime}$ for some $x_{i} \in A$.

$$
\begin{aligned}
\text { iii } \rightarrow \text { i. Obviously, iii } \cdot \rightarrow \text { ii } \cdot \text { And, if }\{x\}^{\prime}=\cup\left\{F_{i}: i \in I\right\}, \\
\quad x=\overline{\{x\}}-\{x\}^{\prime}=\overline{\{x\}}-\cup\left\{F_{i}: i \in I\right\}=\overline{\{x\}} \cap\left\{X-F_{i}: i \in I\right\} .
\end{aligned}
$$

COROLLARY. Each $T^{(m)}$ space is a $T_{0}$ space, and each $T_{0}$ space is a $T^{(m)}$ space for some $m$.

THEOREM 4. An $m$ product of $T^{(m)}$ spaces is again a $T^{(m)}$ space.

Proof. Let $Y=\Pi\left\{X_{i}: i \in I\right\}$ be an $m$-product of $T^{(m)}$ spaces. Then, for each $x \in Y, x=\cap\left\{\pi_{i}^{-1}\left(\pi_{i}(x)\right): i \in I\right\}=\cap\left\{\pi_{i}^{-1}\left[G_{m, i} \cap F_{i}\right]: i \in I\right\}$, where each $F_{i}$ is closed in $X_{i}$ and each $G_{m, i}$ is an intersection of $m$ open sets in $X_{i}$. Thus, $x=\cap\left\{\pi_{i}^{-1}\left[G_{m, i}\right]: i \in I\right\} \cap\left\{\pi_{i}^{-1}\left[F_{i}\right]: i \in I\right\}$, and the continuity of each $\pi_{i}$ yields the desired conclusion. 
THEOREM 5. An $n$ product of $T^{(m)}$ spaces (none of which is $T_{1}$ ) is again $a T^{(m)}$ space if and only if $n \leqq m$.

Proof. The sufficiency follows from the preceeding corollary, while the necessity follows from arguments analogous to those used in the proof of Theorem 1.

REMark. We see thus that there is no $T^{(m)}$ space which inherits this property under arbitrary products with itself. This observation may be employed to construct, for any cardinal $m$, a $T^{(m)}$ space which is not a $T^{(k)}$ space for any $k \leqq m$; we need only take an $m$ fold product of $T_{D}$ spaces, none of which is $T_{1}$.

\section{References}

[1] C. E. Aull and W. J. Thron, Separation axioms between $T_{0}$ and $T_{i}$, Indag. Math. 23 (1962), $26-37$

[2] W. J. Thron, Topological Structures (Holt, Rinehart, and Winston, New York, 1966).

Department of Mathematics

Cleveland State University

Cleveland, Ohio

and

Case Western Reserve University

Cleveland, Ohio 